\title{
Virtual Rotations for Maneuvering in Immersive Virtual Environments
}

\author{
Pauline Bimberg \\ clara.pauline.bimberg@uni-weimar.de \\ Bauhaus-Universität Weimar \\ Weimar, Germany \\ Alexander Kulik \\ kulik@consensive.com \\ Consensive $\mathrm{GmbH}$ \\ Weimar, Germany
}

\author{
Tim Weissker \\ tim.weissker@uni-weimar.de \\ Bauhaus-Universität Weimar \\ Weimar, Germany \\ Bernd Froehlich \\ bernd.froehlich@uni-weimar.de \\ Bauhaus-Universität Weimar \\ Weimar, Germany
}
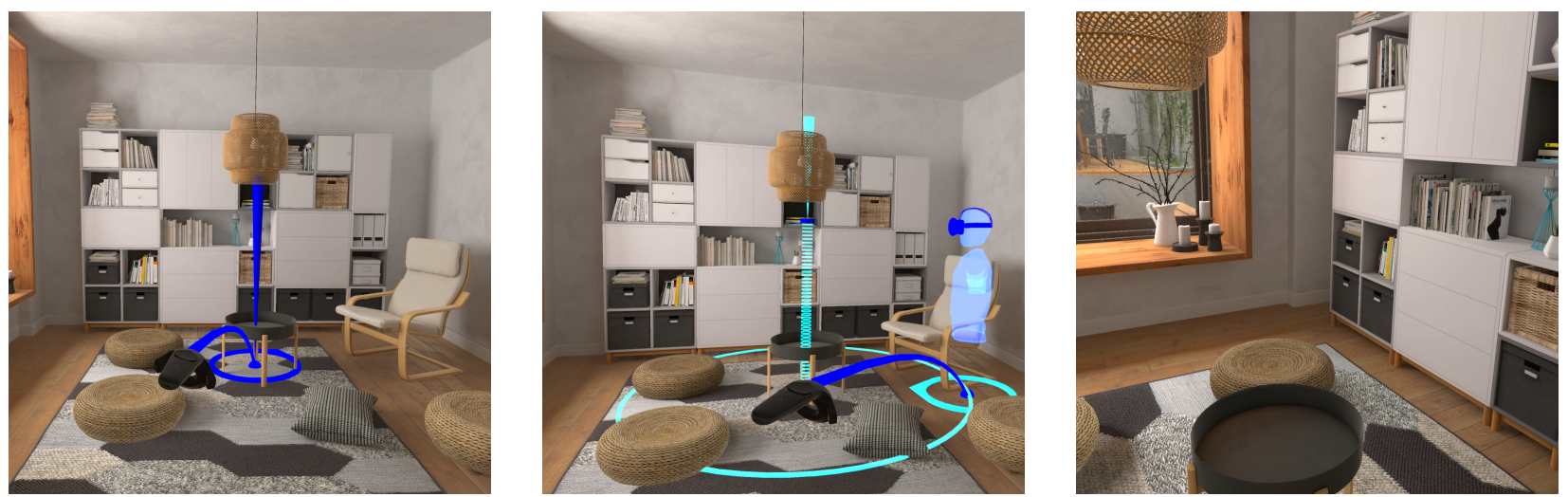

Figure 1: Anchored Jumping allows a user to specify a rotation axis (left) and a new viewing position relative to it (middle). The user will then teleport to the indicated viewing position and automatically face the specified rotation axis (right). Depending on the scenario, this axis can either be re-activated for the next maneuvering step or be re-specified for each jump.

\begin{abstract}
In virtual navigation, maneuvering around an object of interest is a common task which requires simultaneous changes in both rotation and translation. In this paper, we present Anchored fumping, a teleportation technique for maneuvering that allows the explicit specification of a new viewing direction by selecting a point of interest as part of the target specification process. A first preliminary study showed that naïve Anchored fumping can be improved by an automatic counter rotation that preserves the user's relative orientation towards their point of interest. In our second, qualitative study, this extended technique was compared with two common approaches to specifying virtual rotations. Our results indicate that Anchored fumping allows precise and comfortable maneuvering and is compatible with techniques that primarily support virtual exploration and search tasks. Equipped with a combination of such
\end{abstract}

This work is licensed under a Creative Commons

Attribution-NonCommercial-NoDerivs International 4.0 License.

VRST '21, December 8-10, 2021, Osaka, Japan

(C) 2021 Copyright held by the owner/author(s).

ACM ISBN 978-1-4503-9092-7/21/12.

https://doi.org/10.1145/3489849.3489893 complementary techniques, seated users generally preferred virtual over physical rotations for indoor navigation.

\section{CCS CONCEPTS}

- Human-centered computing $\rightarrow$ Virtual reality; User interface design.

\section{KEYWORDS}

Virtual reality, 3D navigation, Target-based travel, Maneuvering, Virtual rotation, Teleportation, Jumping

\section{ACM Reference Format:}

Pauline Bimberg, Tim Weissker, Alexander Kulik, and Bernd Froehlich. 2021. Virtual Rotations for Maneuvering in Immersive Virtual Environments. In 27th ACM Symposium on Virtual Reality Software and Technology (VRST '21), December 8-10, 2021, Osaka, Japan. ACM, New York, NY, USA, 10 pages. https://doi.org/10.1145/3489849.3489893

\section{INTRODUCTION}

Navigation tasks in virtual environments can be classified as exploration, search, or maneuvering [2]. While exploration and search usually entail larger movements through the scene, maneuvering involves rather small translation and rotation movements around a particular object of interest in order to investigate it from different 
perspectives. For head-mounted displays, commonly used shortdistance teleportation techniques (jumping) are not well-suited for this task as they require the user to turn physically after each jump to face the object of interest again. Frequent physical rotations can become exhausting for the user or might be even impossible to perform in certain seated or other spatially confined setups.

To overcome these issues, we analyzed prior approaches to combining jumping techniques with additional options for executing rotations virtually. We observed that earlier work typically extends the target specification phase of jumping with the explicit specification of a new viewing direction [3, 16, 28]. In contrast to this approach, classic maneuvering techniques based on steering or animated transitions typically allow their users to explicitly select a point of interest (POI) in the scene, which defines the viewing directions for any subsequent navigation steps [11,21].

We build on this approach and present Anchored fumping, a novel technique optimized for maneuvering tasks in head-mounted displays that relies on short-distance teleportation instead of steering or animated transitions (Figure 1). With Anchored fumping the user selects a POI first and specifies the actual target location of the jump in a second step. We also suggest adding an automatic counter rotation that preserves the user's relative orientation towards their point of interest and therefore reduces the need for physical rotations. We furthermore suggest the integration of Anchored Jumping with other virtual rotation approaches to cover a broad range of navigation tasks. We examined the use of Anchored fumping in two user studies. In a pilot study using a pre-set POI, we compared Anchored Jumping with and without counter rotations and examined usability and user comfort. In a second study, we compared Anchored Jumping with Free fumping [3] and Snap Rotations for specifying virtual rotations in order to analyze their individual strengths and mutual complementarity. These techniques served as a suitable baseline since they are often seen in commercial applications.

In summary, our research led to the following contributions:

- Anchored fumping, an enhanced teleportation technique for HMDs that allows the explicit definition of a viewing direction by selecting a point of interest as part of the target specification process,

- the results of a pilot study with seated users showing that naïve Anchored fumping can be improved by an automatic counter rotation that preserves the user's relative orientation towards their point of interest, and

- the results of a qualitative user study with seated users indicating that Anchored fumping is especially suitable for solving maneuvering tasks and that its combination with Free fumping and Snap Rotations supports a broad range of use cases.

Overall, the positive user feedback on Anchored fumping for maneuvering tasks as well as its complementarity to other virtual rotation techniques make it a valuable addition to the portfolio of navigation techniques in virtual environments.

\section{RELATED WORK}

\subsection{Navigation in Virtual Environments}

As most virtual environments cannot be overlooked from a single vantage point [25], users require navigation techniques that allow them to change their virtual position and orientation. The three main types of navigation are exploration for the sake of gathering information about the layout and/or content of the virtual world, search for reaching a previously defined goal, and maneuvering for making small precise movements to view an object or feature of interest from different perspectives [2]. Different metaphors can be utilized for the interpretation of user inputs and their transfer to movements in the virtual environment. Overall, the most direct mapping is achieved by physical walking in a tracked real-world space. While this natural approach is often associated with the biggest possible ease of use, comfort, and sense of presence [34], its use is bound to the size of the available tracking space. For larger distances, virtual travel techniques are often provided as an addition.

Steering, one of the most common virtual travel metaphors, lets the user continuously specify the direction and speed of their movements in the virtual world [2]. During this process, users are able to continuously integrate the current visual feedback into their mental map of the environment - a process also known as continuous spatial updating $[5,16]$. The downside of using steering for virtual navigation lies in its tendency to cause symptoms of simulator sickness $[12,29]$. This problem is especially experienced by users of HMDs $[19,29,32]$ and can be partially mitigated through visual aids such as field-of-view modifications [9] or rest frames [4]. A prominent alternative to steering is short-distance teleportation in vista space, also known as jumping, which has shown promising results regarding user well-being and performance $[3,6,36]$. A common concern against this technique is that users might lose their orientation in the virtual environment. However, while the process of continuous spatial updating process might be hindered by the absence of motion cues, there is evidence that users can largely make up for this by either backtracking in the environment [6] or using static visual features such as spatial boundaries and landmarks as piloting cues $[5,16]$. Motivated by its high user comfort, our work in this paper builds upon jumping navigation as the basis for our technique developments in the area of virtual rotations.

\subsection{Maneuvering in 3D Space}

Maneuvering presents users with a different challenge than other virtual navigation tasks. Its main objective is not to cover ground within the virtual environment but to gain new specific perspectives onto an object or feature of interest. Since users within fully tracked systems often utilize physical walking for these small changes, dedicated maneuvering techniques have mainly been developed for 2D screens or projection-based VR systems [2, 21, 23]. A common approach in this regard is letting users select a point around which rotational movements are applied. In some cases, such pivot points can be set anywhere within the virtual space, like it has been suggested by Kulik et al. for the inspection of large scanned 3D models, or be inferred from the users viewing direction as suggested by Trindade and Raposo [23, 33] . Other approaches let users select a virtual object and either center their own movement around the object or 
use it as a lever to move the whole virtual scene around themselves $[1,22]$. More specific metaphors, like the HoverCam technique, automatically guide the user's camera movements around a virtual object's surface [18]. Similarly, the target-based Navidget technique lets users select a specific camera position around a point on the surface of a virtual object [11]. Navidget's extension for the use with tracked input devices in virtual reality allows the specification of a point of interest on the surface of an object via ray casting followed by the selection of the viewpoint by moving a camera widget along a spherical helper geometry shown around it. After ending the specification process, the user is flown towards the target perspective along an automatically generated path [21]

While the methods presented in related work seem to work well for their specific use-cases, research on the adoption of these methods for head-mounted displays and the investigation of their influence on user experience and well-being is sparse. We aim to bridge this gap by comparing the use of typical rotation methods for jumping navigation and proposing a new extension of the jumping metaphor optimized for maneuvering tasks.

\subsection{Virtual Rotations for Navigation}

Common HMD setups allow users to freely rotate their heads and bodies to look around in the virtual environment. As a result, virtual navigation techniques for these systems often only provide capabilities for virtual translations while asking users to rotate their heads physically if required. However, this can be problematic in specific situations. Seated setups, for example, often do not feature the ideal interaction space for full turns. This is especially common in home setups where the user might sit at their desk or even on a couch while being immersed in a virtual world. Combined with the small FOV of most HMDs, this can lead to users favoring a particular primary viewing direction for activities in the virtual environment [13]. For this reason, many design guidelines for virtual experiences recommend setups which either present content preferably in the frontal parts of the scene or actively guide the user's attention from one object of interest to the next [35]. However, these approaches are not suitable for more explorative scenarios without any predefined points of interest and sequences. For these cases, virtual navigation techniques have to be enhanced to allow the explicit user-initiated specification of rotation changes as well. This addition of new degrees of freedom to virtual navigation can be done in different ways and comes with its own challenges regarding comprehensibility and user comfort. Overall, virtual rotations can be specified separately from virtual translations (Section 2.3.1) or be integrated into the travel process by allowing the simultaneous specification of a new position and viewing direction (Section 2.3.2).

2.3.1 Separate Definition of Virtual Rotations. Early research on virtual rotations delivers some evidence that proprioceptive feedback plays a larger role in the integration of rotations than of translations with users finding virtual rotations harder to imagine and real rotations harder to ignore than corresponding translations $[8,20,26]$. On the other hand, Riecke et al. reported that users tend to overestimate the extent of real turns when they are not given any visual feedback [30]. This phenomenon has also been shown by Jaekl et al., who asked users to adjust the rotation of a virtual environment manually to match the user's rotation in the real world [14]. This overestimation has inspired techniques which amplify the rotational results of real head movements in HMDs, making it easier for users to look around in virtual environments during navigation and search [15]. Although it was employed successfully by other researchers, it has also been connected to symptoms of simulator sickness, loss of orientation, and usability deficits [27, 31]. To avoid motion sickness resulting from steering movements, Farmani and Teather suggested discrete rotational jumps to be activated at a certain rotation speed, which led to a decrease in motion sickness while leaving other quality factors unaffected [7]. In a study conducted by Riecke et al., participants were able to understand changes in orientation within a known environment based on two static pictures being shown after each other [30]. In addition, Rahimi et al. could show that users were able to understand rotational jumps in a feature-rich virtual environment [28]. The use of user-controlled rotational jumps or Snap Rotations has also become a common navigation enhancement for commercial VR systems.

While related work shows that separate virtual rotations and translations can be mentally integrated using environmental cues, this two-step approach might become especially bothersome for maneuvering, where both types of transformation are often required at the same time.

2.3.2 Integrating Virtual Rotations and Translations. Several researchers have described travel techniques that allow the simultaneous specification of position and orientation of the user within the virtual space. Bozgeyikli et al., for example, presented an extension of jumping where users could prepare virtual rotations during target specification. While some of their users appreciated the efficiency added by these Free Rotations, most of them complained about feelings of dizziness and disorientation [3]. Contrasting results were reported by Funk et al., who compared the previously described technique to their approach of curved jumping trajectories [10]. Working on a target acquisition task, their users were able to use Jumping with Free Rotations efficiently without indications of a heightened mental load, dizziness, or orientation loss compared to regular jumps. The research of Kelly et al. and Cherep et al. gives us further insights on the influence of the virtual scene on the integration of virtual jumps. In their work, the terms partially concordant and discordant define jumps affecting position only and jumps affecting position and orientation together, respectively. Users generally showed worse spatial orientation when using discordant jumping methods in comparison to partially concordant jumping. This effect could, however, be partially mitigated allowing users to orient themselves using spatial borders within the environment and by adding landmarks to these boundaries $[5,16]$.

These results indicate that users can interpret discordant jumps within an environment that supplies them with the necessary spatial features to regain orientation after a jump. To our knowledge, however, the use of virtual rotation techniques has not yet been investigated in relation to different navigation tasks. We aim to bridge this gap by comparing the advantages and disadvantages of a newly developed specification method in relation to Snap Rotations and jumping with integrated Free Rotations. 


\section{INTERACTION DESIGN}

Building on the results presented by related work (see Section ??) we present an extension of the classic jumping metaphor called Anchored Jumping to support maneuvering in Head Mounted Displays. This usage context motivates some specific requirements that have guided our technique design:

Emphasis on POI selection. Anchored Jumping asks users to explicitly select a POI in the scene first and then follow-up with the selection of the user's new target position. Selecting the targeted POI as a preparation step allows to infer the required change in viewing angle during the selection of a new position automatically instead of requiring manual adjustments by the user to ensure that they will face the POI after the jump. Furthermore, an explicitly selected POI can be kept for multiple jumps and therefore simplify future target specification processes.

Instantaneous transitions. While target-based navigation has been combined with slow or sped-up continuous transitions in previous work to retain spatial awareness, we decided to use instantaneous over continuous transitions which have been linked to higher comfort scored of HMD users [24]. This trade-off has been motivated by positive results regarding spatial updating performances when using jumping with instantaneous transitions in feature-rich indoor spaces $[5,6,16]$.

Restriction to yaw rotations. Several prior implementations of maneuvering techniques allow the specification simultaneous changes in all six degrees of freedom of a user's viewing perspective. This expressiveness can come with several undesired side effects that are especially pronounced in head-mounted displays. First, abrupt changes in user height or a misalignment of the virtual and physical ground plane might lead to unpleasant sensory conflicts between the experience of gravity by the vestibular system and the expectations induced by visual feedback [29]. Second, simultaneous changes around multiple rotational axes were linked to increases in spatial disorientation and motion sickness [17]. As a result, we designed Anchored fumping to provide ground-based navigation while inducing virtual rotations only around the upright axis.

Seamless integration. As reported by related work, maneuvering is only one of three tasks a user might face when navigating through a virtual space [2]. We therefore designed Anchored fumping to serve as an extension of the regular jumping process without changing the target definition process for regular jumps that do not feature any rotational changes. We furthermore suggest the combination of Anchored fumping with other known rotation techniques for jumping that are more suitable for exploration and search tasks.

\subsection{Anchored Jumping}

Following the typical two-step process of existing maneuvering techniques, Anchored fumping lets the user specify a center of rotation, which then becomes the anchor for the viewing direction when specifying the actual target position of the user in a second step (Figure 1). To ensure a seamless integration into the regular jumping process, the rotation axis is specified in a similar way as the target in conventional jumping implementations (Figure 1 left). After the user has confirmed the position of the rotation axis, an additional preview avatar allows for the specification of the user's target position. If the user lets go at this stage of the jumping process, they can trigger a regular jump without rotation changes. By moving the parabolic pointing ray away from the rotation axis and outside of a set safety radius of $0.3 \mathrm{~m}$, the viewing direction of the user's future position is locked onto the axis which is visualized as a geometry in the scene (Figure 1 middle). The introduction of the safety zone ensures the technique's compatibility with regular jumping while also avoiding the large impact that small movements close to the POI would have on the specified viewing direction. After triggering an Anchored fump, users are instantly teleported to their specified destination and rotated to face the POI. To provide the user with a visual connection between their previous and current position, the axis is not hidden directly upon triggering the virtual jump but progressively fades out within the next 0.5 seconds. Depending on the application scenario, the defined axis can either only exist for one definition step or be reused as a static rotation anchor for several consecutive jumps until it is explicitly discarded. Applications that are particularly focused on presenting specific objects could also predefine rotation axes that are activated when the user stands near them. In our research for this paper, we investigated the use of a pre-defined and static rotation axis in a first preliminary study (Section 4) and the process of repeating the complete interaction sequence for each anchored jump in our second, mainly qualitative user study (Section 5).

\subsection{Counter Turns}

So far, we have assumed that the user always looks directly in the direction of the rotation axis after performing an Anchored fump. This straightforward approach, however, can lead to a few unintended side effects when a user performs multiple jumps around an object. During the definition process for a new jump destination, the user often has to turn their gaze away from the point of interest (POI) towards their new target position. If the following teleport simply orients the user towards their point of interest, their relative orientation towards their viewing target changes. In this case, another jump into the same direction would cause the user to turn even further away from their starting direction, which usually leads to their body turning along. As a result, a sequence of jumps might require the user to rotate their body several times, which needs more physical space and can lead to discomfort. The initial definition of the POI by the user allows for an alternative to this mapping, which we call Anchored Jumping with Counter Turns as opposed to Direct Turns. The idea is to neutralize the head's turn away from the POI while specifying the destination of the jump by adding a corresponding rotation offset to the head orientation after the jump (Figure 2). This requires the user to simply turn back towards the object of interest and thus allows them to jump around objects while keeping their interactions centered within a limited angular range in the real-world space. This feature is especially important for users who are seated in non-rotating chairs and for those who need to face a certain direction to avoid losing tracking in a constrained tracking setup or getting tangled in cables. 

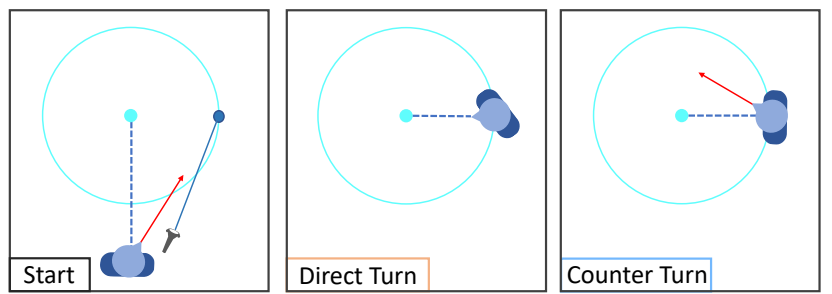

Figure 2: Illustration of the effects that the two variants of Anchored Jumping have on the spatial relations between user and point of interest.

\subsection{Combining Anchored Jumping with Alternative Virtual Rotations Techniques}

Anchored Jumping was designed to support maneuvering tasks and can be combined with other approaches for specifying virtual rotations that do not rely on the definition of a point of interest. As a result, we propose a set of complementary virtual rotation techniques that can be selected and operated with a single controller. Besides Anchored fumping, we suggest adding jumps with fully integrated direction changes (Free fumping) and the possibility to turn on the spot in fixed increments (Snap Rotation).

For our Free fumping technique, we decided to follow the mapping described by Bozgeyikli et al., which lets the user change the orientation of their target position by rotating their controller around its roll axis [3]. Our implementation maps a one-degree turn of the controller to a two-degree turn of the target indicator in the corresponding direction. Free fumping is used instead of Anchored fumping as long as the target position stays within the small safety zone around the POI as described in Section 3.1. Snap Rotations of thirty degrees to the left and to the right can be triggered as an alternative to Anchored and Free Jumping by simple button presses.

For our user studies, the different rotation techniques were implemented using the HTC Vive controller. In our selected button mapping for Anchored and Free fumping, slightly touching the controller's trigger button activates the target ray while fully pressing it confirms the selected position. The subsequent button release triggers an instantaneous jump to the specified target position. Snap Rotations to the left or right are activated by clicking the touchpad button of the controller on the corresponding side.

\section{PRELIMINARY USER STUDY}

Our first preliminary user study investigated the influence of added Counter Turns on the use and user reception of Anchored fumping.

\subsection{Study Design}

Our preliminary study was carried out as a within-subjects study with two conditions, one using Anchored fumping with Direct Turns and one with Counter Turns. All studies were carried out in compliance with current health and safety regulations.

4.1.1 Technical Setup and Study Environment. To interact with the virtual environments, participants of our study used an HTC Vive Pro head-mounted display and one controller. Since virtual rotations are especially useful for seated setups (see Section 2.3), participants sat on a swivel chair that was tracked by an attached tracking target. To prevent users from getting caught in the cables of the HMD, they were mounted on the ceiling of the study room. The scene used for our pilot study was constructed and rendered using the Unity3D engine. It consisted of a single room with some objects, two windows and a doorway. A depiction of the study environment from a user's perspective can be found in Section 5 .

4.1.2 Study Task. Each study condition consisted of two sets of tasks, one using a small focus object and one using a large focus object which users should inspect from different viewing angles. To do this, they were asked to jump to indicated positions around the respective focus object and to look at it from their new vantage point. The target positions were placed on a radius of $1.5 \mathrm{~m}$ around the small and $2 \mathrm{~m}$ around the large object.

There were nine different target positions for each object with one task being an initial jump directly towards the target and eight further tasks asking for turns of 30,45, 90, or 105 degrees around the object from the last position. The sub-tasks were presented in a randomized order. To compare the effects of the Direct Turn and the Counter Turn in isolation, we used one of the simplification methods for Anchored Jumping described at the end of Section 3.1. Here, the first stage of the definition process was excluded by setting a static rotation anchor to which all jumps were automatically bound to.

4.1.3 Study Sequence and Measures. After users gave their informed consent, the experiment started with a verbal explanation of both study conditions, which were then presented in a counterbalanced order. Each of the study conditions consisted of a free exploration phase, where users could learn the technique and ask questions, five unrecorded tutorial tasks, and finally the two recorded task sequences described in Section 4.1.2. To keep track of the users' well-being during our study, we asked them for a discomfort score after the completion of the tutorial as well as after each set of tasks. The discomfort score was measured by asking users the following question: "On a scale of $0-10,0$ being how you felt coming in and 10 is that you want to stop, where are you now?" $[9,29]$. After the last task of each study condition, users were additionally asked to rate how often they felt confused after a jump in the virtual environment on a scale of 0 to 10 . Participants were then given the opportunity to take a break from wearing the HMD and, when they felt ready to continue, completed the same study sequence for the second condition. At the end of the study, users were interviewed about their overall preference when it came to the two presented methods as well as their opinion on the user experience. After the successful completion of the study, which took about 30min in total, participants were rewarded with an expense allowance of 5 Euros.

\subsection{Results}

The sample of our first study consisted of 8 participants, two of which were women. They were between 23 and 45 years of age ( $M=29.5$ years, $M e d=27$ years). In adherence to the local health and safety guidelines, the study sample was restricted to faculty members and students of our university.

Figure 3 plots the angular range of physical rotations performed by each user in each condition, which was measured by recording 
the forward direction of the head-mounted display in the tracking space for each frame. The average angular extent covered by the users' movements was 110.62 degrees for Counter Turns $($ Med $=$ $\left.110^{\circ}, \sigma=35.36^{\circ}\right)$ and mostly centered around the initial viewing direction. In the Direct Turn condition, an average angular extent of 325.75 degrees $\left(\mathrm{Med}=342.0^{\circ}, \sigma=43.16^{\circ}\right)$ was covered. In particular, all users performed continuous turns around their body axis with half of the users requiring more than one full rotation to solve the task.

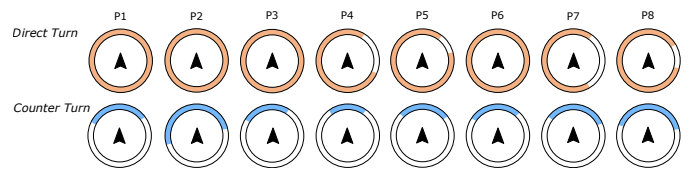

Figure 3: Illustration of the amount of physical rotation performed in our pre-study with each column representing a participant in the Direct Turn (top row) and Counter Turn condition (bottom row). The arrows indicate the starting orientation of the HMD in the physical space while the colored circle segments show all forward directions that occurred during the course of the respective condition.

The average maximum difference between the users' head direction and the forward direction of the chair was measured at 102.58 degrees for the Direct Turn (Med $\left.=102.08^{\circ}, \sigma=32.81^{\circ}\right)$ and at 49.40 degrees for the Counter Turn condition $\left(\right.$ Med $\left.=48.35^{\circ}, \sigma=10.33^{\circ}\right)$. The confusion scores given by the users after each condition averaged at 3.5 of 10 for the Direct Turn (Med $=2.5, \sigma=3.34$ ) and 0.75 out of 10 for the Counter Turn condition (Med $=0.0, \sigma=1.16$ ). Throughout the experiment, our users reported average discomfort scores of 1.04 out of 10 points for the Direct Turn condition (Med $=0.5, \sigma=0.74)$ and 0.62 out of 10 points for the Counter Turn condition (Med $=0.5, \sigma=1.60) .6$ of 8 users generally preferred the Counter Turn condition with one of the remaining two users remarking that they would probably prefer it in a more open scenario but did not need it during the study. The remaining participant remarked that they preferred the Direct Turn because looking directly at the target after a jump matched their expectations of their future orientation. When asked about their comfort level during navigation, all but one user reported that they felt more comfortable when using the Counter Turn method.

\subsection{Discussion}

While our sample for the preliminary study was not large enough to warrant the use of statistical tests, we can draw some preliminary conclusions from the reported data. Overall, the addition of the Counter Turn had the intended effect on rotation behavior by centering the users' turns around their initial forward direction and preventing them from having to perform full revolutions within their tracking space. The heightened user comfort that was reported for the counter turns in our spatially unrestricted setup could be explained by the angular differences between the users' head and chair rotations, which went up to an average of 102.58 degrees when using Direct Turns. We also noted that the integration of translation and rotation changes into one (discordant) jump did not seem to lead to disorientation in our pre-study, especially when the Counter Turn method was active. This could indicate that the planning process as well as the binding of the turns to a visible feature in the environment supplied users with enough context to mentally process their new positions within the environment.

\section{SECOND USER STUDY}

Since the ongoing global pandemic prevented us from carrying out a user study with a large participant sample, we decided on an exploratory study design with an emphasis on qualitative measures. Based on related work and the preliminary findings of our pre-study, we compared the use of the Anchored fumping technique with Counter Turns to the use of Free fumping and to the combination of partially concordant jumps and Snap Rotations. To gather data on the whole definition process of Anchored fumping and to ensure compatibility between the three conditions, we decided to ask our users to define a new rotation axis for every Anchored fump instead of providing a mechanism for keeping the rotation axis static as it was done in the first user study.

Keeping in mind our goal to design a technique that would specifically address the challenge of maneuvering for jumping navigation while being compatible with other rotation mechanisms, our second study design was guided by the following research questions:

RQ1 How do the three virtual rotation techniques influence performance in a controlled maneuvering task?

RQ2 Can the suggested combination of all three virtual rotation techniques be easily operated and understood?

RQ3 Which scenarios benefit from the use of each of the three virtual rotation techniques?

To address all of these aspects with a limited study sample, our study design consisted of two phases: one controlled study scenario similar to our preliminary study (Section 5.1) and one more open usage scenario (Section 5.2).

The overall study protocol resembled that of the first study, beginning with the users signing a consent form and ending with the completion of the free study scenario. The whole study took around 60 minutes to complete and participation was rewarded with an expense allowance of 10 Euros. A description of the technical setup that was employed in both study scenarios and the virtual study environment that was used in the controlled study scenario can be found in Section 4.1.1.

\subsection{Controlled Study Scenario}

5.1.1 Study Task. Participants performed the Anchored Fumping, Free fumping and Snap Rotation conditions in a counterbalanced order. Each condition consisted of four sets of tasks that appeared in randomized order, two using small and mostly symmetric focus objects and two using larger rectangular focus objects. Around each of these objects, users were asked to complete six placement tasks and look towards the object from the respective positions. To ensure that users were able to both interact with the object and look at it directly from the goal position, we added a sphere geometry between the focus object and the target cone. After performing a jump to the target position, users were asked to touch the sphere, which then faded away to reveal a word that had to be read out aloud. A visualization of the task process can be found in Figure 4. 
The tasks assigned to each of the objects were comprised of jumps in both rotational directions with individual tasks asking users to perform jumps with turns of 45, 90 and 180 degrees. The sequence of these tasks was randomized, but it was ensured that each type of task appeared with identical frequencies in each study condition.
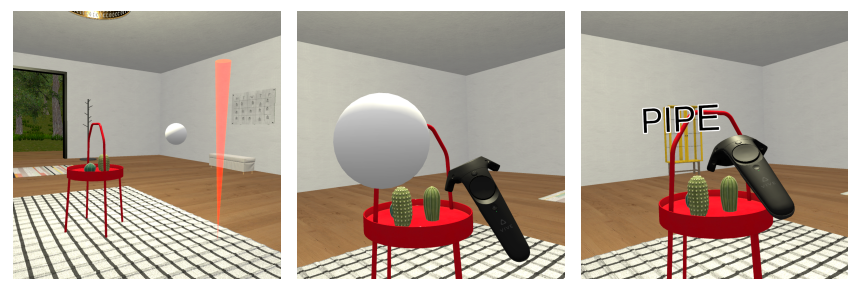

Figure 4: The process of a single task item in our study, which starts by jumping to the position of the target cone (shown on the left) and then asks the user to turn towards the target sphere (middle) and touch it to reveal a randomly selected word (right) to be read aloud.

5.1.2 Study Sequence and Measures. The three conditions were presented in counterbalanced order. Each condition consisted of a free try-out stage, five unrecorded tutorial tasks, and the four recorded task sets as described in Section 5.1.1. The discomfort scores were collected after the tutorial, at the halfway point of the recorded tasks, and after the completion of all recorded tasks in each condition. At the end of each condition, users were asked for their confusion scores and given the opportunity to take a break from wearing the HMD. Having completed all three study conditions, users were asked to take off the HMD and rest for at least 5 minutes until they were comfortable to continue with the free study scenario.

\subsection{Free Study Scenario}

The virtual environment for this part of the study consisted of a highly detailed indoor environment ${ }^{1}$, which can be seen in Figures 1 and 5 .

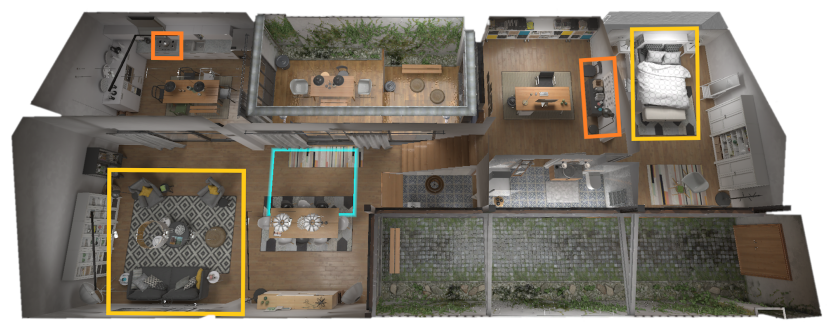

Figure 5: The virtual environment used for our second study scenario. The turquoise square marks the user's starting point in the virtual scene. The yellow and orange squares mark the features that users were asked to inspect in more detail.

${ }^{1}$ https://assetstore.unity.com/packages/3d/environments/urban/archvizpro-interiorvol-6-120489
Since the goal of the second study part was to investigate how participants would use the three techniques in an open scenario, the main task was to explore the whole virtual environment and to form an opinion about the combination of all available techniques for defining virtual rotations. After users had explored the house and tested all techniques to their own satisfaction, they were asked to navigate to four different rooms and fulfill one task in each of them that was derived from typical maneuvering scenarios. By employing this mix of free exploration, task-driven search and maneuvering, we ensured that all three main use cases of navigation had been addressed by each user. After fulfilling the tasks in the virtual environment, they took off the HMD for a qualitative interview.

\section{RESULTS}

The sample of our study consisted of 12 participants, four of which were women. They were between 23 and 45 years of age $(M=28.0$ years, $M e d=27.5$ years) and were selected according to the criteria described in Section 4.2 .

\subsection{Quantitative Measures}

The average time taken for the target specification of a jump was 5.47s for Anchored Jumping ( $M e d=5.22 s \sigma=1.41 \mathrm{~s})$, 4.82s for Free fumping (Med $=4.59 \mathrm{~s} \sigma=1.65 \mathrm{~s})$, and 3.21s for Snap Rotations $(\mathrm{Med}=3.01 \mathrm{~s}, \sigma=1.22 \mathrm{~s})$. The average subsequent times between the execution of the jump and users touching the sphere were measured at 1.12s for Anchored fumping ( Med $=1.06 \mathrm{~s}, \sigma=0.36 \mathrm{~s}$ ), $1.40 \mathrm{~s}$ for Free Jumping (Med $=1.31 \mathrm{~s}, \sigma=0.49 \mathrm{~s}$ ), and 2.53s for Snap Rotations ( $\mathrm{Med}=1.98 \mathrm{~s}, \sigma=0.95 \mathrm{~s})$. The distributions of these durations are visualized in Figure 6 (left).

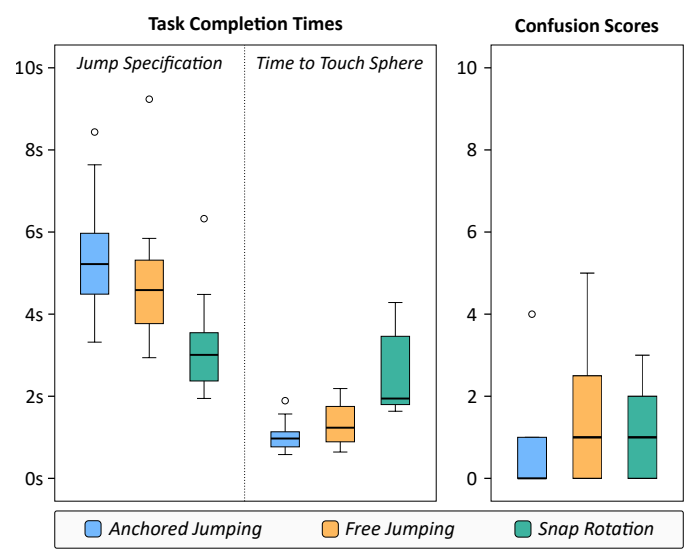

Figure 6: Boxplots showing the time users took to specify a jump and to subsequently touch the target sphere (left) as well as the confusion scores that they reported after each condition (right).

The confusion scores given after each condition averaged at 0.67 out of 10 for Anchored Jumping (Med $=0.0, \sigma=1.16$ ), 1.42 for Free fumping (Med $=1.0, \sigma=1.73$ ), and 1.08 for Snap Rotations $(\mathrm{Med}=1.0, \sigma=1.24)$. The distribution of these scores is visualized in Figure 6 (right). The mean value of the three measurements of discomfort per condition averaged at 0.92 for Anchored fumping 
$($ Med $=0.5, \sigma=1.12), 0.69$ for Free fumping $($ Med $=0.17, \sigma=0.94)$, and 1.22 for Snap Rotations (Med $=0.5, \sigma=1.71$ ).

\subsection{Interview Answers}

The following section summarizes the remarks that were made during our qualitative interview. Numbers behind statements indicate how many participants referred to a certain aspect in their interview.

\subsubsection{Snap Rotation.}

Advantages. When asked about the positive qualities of Snap Rotations, users appreciated the ease of use and simplicity of the technique, calling it "straight forward", "easy", "simple", "fast", and "intuitive" (5). Users also remarked that they had "nothing to think about" and that they felt "no confusion" (2). Three users mentioned that they already knew the technique from other applications or toolsets. One user remarked that they felt safer using Snap Rotations since they did not have to turn their chair to look around.

Disadvantages. Negative aspects were the amount of time (2) and effort (2) that this method took for larger turns. Two users found the technique annoying and three users found it uncomfortable. Four users also mentioned the lack of precision of this technique due to the fact that the rotation angle was fixed to $30^{\circ}$.

Use Cases. When asked about situations in which they employed Snap Rotations, participants mentioned situations in which they wanted to make fast movements (2) or smaller, often correctional changes (4). The most-named application case was looking around while staying in the same position (5), especially in corners where there is not enough space to set a new target position (3). Only one user never used the Snap Rotations in the free study scenario since they found it too annoying.

\subsubsection{Free Jumping.}

Advantages. Commenting on the Free fumping technique, users generally reported that they found it intuitive and easy to use (5) and liked it especially for defining smaller changes in direction (3). One user remarked that they could achieve precise positioning with the technique while the rotation definition worked "well enough".

Disadvantages. Three users reported that they needed to get used to the technique and were sometimes confused by their change in orientation. Other users found the defined rotations to be "not precise" (2) or "difficult to get right" (1). One user mentioned that it was difficult for them to control rotations and translations at the same time. Seven users considered the technique to be uncomfortable to use in some situations. Two users especially named larger turns as difficult, and two others found the technique to be awkward when selecting positions at their side or in close range.

Use Cases. Free fumping was deemed suitable for the definition of small (3) and fast (3) changes or minor adjustments to the rotation after a previous jump. The technique was used mostly for "coarse, imprecise" (2) or "general" changes in direction or to look in a general direction and not at a target (3). One user each named "small adjustments around an object" and wanting to look at "something on a wall" as application cases for the Free fumping technique.
Five users named the "free exploration of rooms" and "general navigation" as use cases for such turns.

\subsubsection{Anchored Jumping.}

Advantages. The Anchored fumping technique was appreciated by eight users for its precision when wanting to look at a certain object in the environment. Three users commented on the technique as being "convenient", "less confusing", or putting the "least amount of stress" on them. One user also remarked that the technique was "fast and easy" after they had learned to use it.

Disadvantages. Several users remarked that they needed a little more time to understand the technique when compared to the others (4) with some commenting that changing the definition process from thinking mostly about the position they wanted to stand in to thinking about the position that they wanted to look at was difficult to them (3). One user did not find the technique helpful for small adjustments, and one user remarked that the movement range of the technique was reduced due to them having to place the rotation axis.

Use Cases. When listing use cases for the Anchored fumping technique, all users mentioned situations where they wanted to "directly look at" or "inspect" a certain object of interest. Only one user remarked that they had used both the Anchored fumping and the Free fumping method for this task and preferred Free fumping because of its simplicity. Another user mentioned that they used the technique when wanting to make large turns, which were uncomfortable or annoying to define with the two other techniques. Two users mentioned that they would like to use Anchored fumping specifically in museum contexts to "inspect artifacts" or to position themselves in relation to an object of interest without incidentally walking into someone.

6.2.4 Combination of Techniques. Commenting directly on the combination of the three techniques, users appreciated the freedom and flexibility to choose the rotation technique matching their needs (3). Users also liked that they only needed two buttons for the definition of all rotation changes (2) and generally considered the set to be very useful (5) and "rather intuitive". Two users mentioned that it took them a little practice to use it (2) with one user naming the switch between the definition processes of Anchored and Free fumping as the main difficulty. All but one user remarked that they found use cases where they used each of the techniques with some mentioning that they mostly used a combination of Snap Rotations and Free fumping for their explorations while turning to Anchored fumping for the given maneuvering tasks (3). One person mentioned that they did not need Snap Rotations while another user mentioned that they preferred to only use the Free fumping method. Seven users did not report on any conflicts between the techniques, and three users mentioned small conflicts that could be dealt with easily or avoided with practice. One user reported that they did not really experience any conflicts since they used the Free fumping technique almost exclusively. When users were asked if they would change anything about the integration of the techniques, nine users stated that they would generally leave all of the techniques available at the same time with three remarking that it could be a use-case dependent decision in more simple scenarios. 
Two users suggested making the switch between Anchored fumping and Free fumping more explicit, and one user commented that they did not like the Snap Rotation technique and would leave it out completely.

\subsection{Discussion}

Taking into account both the quantitative measures gathered in our controlled study scenario and the qualitative data gathered in the final interview, we can draw some preliminary conclusions with respect to our three research questions. When asking about each technique's appropriateness for maneuvering tasks (RQ1), the feedback for Anchored Jumping seemed to fulfill our expectations. Most users preferred it for the controlled task scenario and used it when they wanted to look directly at an object in the free scenario. While Free fumping was deemed usable for maneuvering tasks and liked for its simplicity, most users found Snap Rotations annoying and tedious when offered as the only option for maneuvering. Although quantitative measures show that the specification of jumps without integrated turns took the least amount of time, the observed dissatisfaction with Snap Rotations motivates the use of integrated techniques for any task that requires simultaneous changes in position and rotation. According to our quantitative measures, participants took more time for the specification of $\mathrm{An}$ chored fumps, but they needed the least amount of time to touch the target sphere after a virtual jump and reported the lowest confusion scores for this technique. This indicates a trade-off between work invested in the definition process and the precise definition of the target position which was also reflected in comments praising the technique's usability. To benefit from the precision introduced by Anchored Jumps while achieving a similar ease of use as Free fumping, the repeated use of a defined rotation axis should be considered in maneuvering-heavy scenarios. When talking about individual use cases for the three different techniques (RQ3), both Free fumping and Snap Rotations were employed for different purposes. The strength of Free fumping seems to lie in its simple and fast definition process for coarse adjustments during exploration while its limitations were the lower degree of precision and the resulting higher degree of confusion. This problem has also been mentioned by related work [3], but it seems to have been partially mitigated by the visual richness of the virtual scene of our free study scenario. As some users reported on experiencing some strain when using their wrist for the definition of larger virtual rotations, future applications should investigate a dynamic gain instead of a constant amplification factor to enable small precise and large effortless rotations. While most users did not like to use Snap Rotations exclusively, they were applied to look around or to navigate out of tight corners in the environment, which makes them a useful addition to the other rotation techniques. In conclusion, all three techniques for specifying virtual rotations had their individual use cases in the free study scenario with Anchored fumping mostly being used for maneuvering and for the detailed inspection of room features. Free fumping and Snap Rotations, on the other hand, enabled users to freely explore the environment as a whole. This complementarity was highly appreciated and left a rather positive impression concerning the usability of all techniques together (RQ2).
Generally, the observed user behavior and feedback imply that the problems that often lead to the avoidance of virtual rotation techniques in implementations for free navigation did not apply as heavily in our study's feature-rich indoor scenarios as they might do in more spacious virtual environments. This result is in accord with related work on the spatial integration of discordant jumps, including virtual changes to both position and rotation $[5,16]$. Furthermore, none of our users reverted to the exclusive use of physical rotations after they had used our set of virtual rotation techniques even though they were seated in an easy-to-turn swivel chair.

\section{CONCLUSION AND FUTURE WORK}

In this paper, we introduced Anchored fumping for effective maneuvering using short-range teleportation. Our technique allows users to explicitly select a point of interest in the virtual environment to specify the intended viewing direction after a virtual jump. In addition, we showed how Anchored fumping can be seamlessly combined with other common rotation techniques for exploration and search tasks. From our preliminary study, we conclude that adding counter rotations to Anchored fumping is highly beneficial for reducing the required body rotations. From our second study, we conclude that Anchored fumping allows precise placements with respect to objects of interest and seamlessly complements Free fumping and Snap Rotations. Even though users were seated on fully rotating swivel chairs in our studies, all of them preferred the use of our integrated set of virtual rotation techniques over performing physical turns, which indicates that they are a valid alternative to physical rotations.

Because we were not able to carry out a large enough user study for statistical analyses, further research is needed to verify the applicability of our preliminary results to a broader user base. Such further research could also investigate the difference between standing and seated usage scenarios and the influence of different virtual environments in order to make further recommendations on when virtual rotations should be employed. With some of our users specifically mentioning museum contexts as an application scenario of Anchored fumping, it would be interesting to see if virtual maneuvering changes the behavior of visitors of virtual museums, for example if it encourages them to investigate objects from different sides. When only a few important artifacts are present in such a scenario, the trade-off between the freedom of letting the users set arbitrary points of interest and simply choosing from a pre-defined set of anchor axes chosen by the curator could be investigated. Finally, with the rising popularity of social VR systems, it is further relevant to study how Anchored fumping can be used for group navigation in multi-user settings.

\section{ACKNOWLEDGMENTS}

Our research received funding from the Thuringian Ministry for Economic Affairs, Science, and Digital Society under grant 5575/105 (MetaReal). We would like to thank the members of the Virtual Reality and Visualization Research Group at Bauhaus-Universität Weimar (http://www.uni-weimar.de/vr) 


\section{REFERENCES}

[1] D. A. Bowman, D. Koller, and L. F. Hodges. 1997. Travel in immersive virtual environments: an evaluation of viewpoint motion control techniques. In Proceedings of IEEE 1997 Annual International Symposium on Virtual Reality. 45-52. https://doi.org/10.1109/VRAIS.1997.583043

[2] Doug A. Bowman, Ernst Kruijff, Joseph J. LaViola, and Ivan Poupyrev. 2001. An Introduction to 3-D User Interface Design. Presence: Teleoperators and Virtual Environments 10, 1 (2001), 96-108. https://doi.org/10.1162/105474601750182342

[3] Evren Bozgeyikli, Andrew Raij, Srinivas Katkoori, and Rajiv Dubey. 2016. Point \& Teleport Locomotion Technique for Virtual Reality. In Proceedings of the 2016 Annual Symposium on Computer-Human Interaction in Play (Austin, Texas, USA). 205-216. https://doi.org/10.1145/2967934.2968105

[4] Z. Cao, J. Jerald, and R. Kopper. 2018. Visually-Induced Motion Sickness Reduction via Static and Dynamic Rest Frames. In 2018 IEEE Conference on Virtual Reality and 3D User Interfaces (VR). 105-112. https://doi.org/10.1109/VR.2018.8446210

[5] Lucia A Cherep, Alex F Lim, Jonathan W Kelly, Devi Acharya, Alfredo Velasco, Emanuel Bustamante, Alec G Ostrander, and Stephen B Gilbert. 2019. Spatial cognitive implications of teleporting through virtual environments. PsyArXiv (2019). https://doi.org/10.31234/osf.io/cx9vt

[6] Chris G Christou and Poppy Aristidou. 2017. Steering versus teleport locomotion for head mounted displays. In International Conference on Augmented Reality Virtual Reality and Computer Graphics. Springer, 431-446. https://doi.org/10. 1007/978-3-319-60928-7_37

[7] Yasin Farmani and Robert J. Teather. 2020. Evaluating discrete viewpoint control to reduce cybersickness in virtual reality. Virtual Reality (13 January 2020) https://doi.org/10.1007/s10055-020-00425-x

[8] Martin J Farrell and Ian H Robertson. 1998. Mental rotation and automatic updating of body-centered spatial relationships. Fournal of Experimental Psychology: Learning, Memory, and Cognition 24, 1 (1998), 227. https://doi.org/10.1037/02787393.24.1.227

[9] Ajoy S Fernandes and Steven K Feiner. 2016. Combating VR sickness through subtle dynamic field-of-view modification. In 2016 IEEE Symposium on 3D User Interfaces (3DUI). 201-210. https://doi.org/10.1109/3DUI.2016.7460053

[10] Markus Funk, Florian Müller, Marco Fendrich, Megan Shene, Moritz Kolvenbach, Niclas Dobbertin, Sebastian Günther, and Max Mühlhäuser. 2019. Assessing the Accuracy of Point \& Teleport Locomotion with Orientation Indication for Virtual Reality Using Curved Trajectories. In Proceedings of the 2019 CHI Conference on Human Factors in Computing Systems (Glasgow, Scotland Uk). 1-12. https: //doi.org/10.1145/3290605.3300377

[11] M. Hachet, F. Decle, S. Knodel, and P. Guitton. 2008. Navidget for Easy 3D Camera Positioning from 2D Inputs. In 2008 IEEE Symposium on 3D User Interfaces. 83-89. https://doi.org/10.1109/3DUI.2008.4476596

[12] Lawrence J. Hettinger, Kevin S. Berbaum, Robert S. Kennedy, William P. Dunlap, and Margaret D. Nolan. 1990. Vection and Simulator Sickness. Military Psychology 2, 3 (1990), 171-181. https://doi.org/10.1207/s15327876mp0203_4

[13] Yang Hong, Andrew MacQuarrie, and Anthony Steed. 2018. The Effect of Chair Type on Users' Viewing Experience for 360-Degree Video. In Proceedings of the 24th ACM Symposium on Virtual Reality Software and Technology (Tokyo, Japan) Article 30, 11 pages. https://doi.org/10.1145/3281505.3281519

[14] Philip M Jaekl, Robert S Allison, Laurence R Harris, Urszula T Jasiobedzka, Heather L Jenkin, Michael R Jenkin, James E Zacher, and Daniel C Zikovitz. 2002. Perceptual stability during head movement in virtual reality. In Proceedings IEEE Virtual Reality 2002. 149-155. https://doi.org/10.1109/VR.2002.996517

[15] Caroline Jay and Roger Hubbold. 2003. Amplifying Head Movements with HeadMounted Displays. Presence: Teleoperators and Virtual Environments 12, 3 (2003), 268-276. https://doi.org/10.1162/105474603765879521

[16] Jonathan W Kelly, Alec G Ostrander, Alex F Lim, Lucia A Cherep, and Stephen B Gilbert. 2020. Teleporting through virtual environments: Effects of path scale and environment scale on spatial updating. IEEE Transactions on Visualization and Computer Graphics 26, 5 (2020), 1841-1850. https://doi.org/10.1109/TVCG. 2020.2973051

[17] Behrang Keshavarz and Heiko Hecht. 2011. Axis Rotation and Visually Induced Motion Sickness: The Role of Combined Roll, Pitch, and Yaw Motion. Aviation, space, and environmental medicine 82 (11 2011), 1023-9. https://doi.org/10.3357/ ASEM.3078.2011

[18] Azam Khan, Ben Komalo, Jos Stam, George Fitzmaurice, and Gordon Kurtenbach 2005. HoverCam: Interactive 3D Navigation for Proximal Object Inspection. In Proceedings of the 2005 Symposium on Interactive 3D Graphics and Games (Washington, District of Columbia) (I3D '05). Association for Computing Machinery, New York, NY, USA, 73-80. https://doi.org/10.1145/1053427.1053439

[19] Kwanguk Kim, M Zachary Rosenthal, David J Zielinski, and Rachael Brady. 2014. Effects of virtual environment platforms on emotional responses. Computer methods and programs in biomedicine 113, 3 (2014), 882-893. https://doi.org/10. 1016/j.cmpb.2013.12.024

[20] Roberta L. Klatzky, Jack M. Loomis, Andrew C. Beall, Sarah S. Chance, and Reginald G. Golledge. 1998. Spatial Updating of Self-Position and Orientation During Real, Imagined, and Virtual Locomotion. Psychological Science 9, 4 (1998)
293-298. https://doi.org/10.1111/1467-9280.00058

[21] Sebastian Knödel, Martin Hachet, and Pascal Guitton. 2008. Navidget for Immersive Virtual Environments. In Proceedings of the 2008 ACM Symposium on Virtual Reality Software and Technology (Bordeaux, France). 47-50. https: //doi.org/10.1145/1450579.1450589

[22] A. Kulik. 2009. Building on Realism and Magic for Designing 3D Interaction Techniques. IEEE Computer Graphics and Applications 29, 6 (November 2009), 22-33. https://doi.org/10.1109/MCG.2009.115

[23] Alexander Kulik, André Kunert, Stephan Beck, Carl-Feofan Matthes, Andre Schollmeyer, Adrian Kreskowski, Bernd Froehlich, Sue Cobb, and Mirabelle D'Cruz. 2018. Virtual Valcamonica: Collaborative Exploration of Prehistoric Petroglyphs and Their Surrounding Environment in Multi-User Virtual Reality. Presence: Teleoperators and Virtual Environments 26, 3 (2018), 297-321. https: //doi.org/10.1162/pres_a_00297

[24] Daniel Medeiros, Eduardo Cordeiro, Daniel Mendes, Maurício Sousa, Alberto Raposo, Alfredo Ferreira, and Joaquim Jorge. 2016. Effects of Speed and Transitions on Target-Based Travel Techniques (VRST '16). Association for Computing Machinery, New York, NY, USA, 327-328. https://doi.org/10.1145/2993369.2996348

[25] Daniel R. Montello. 1993. Scale and multiple psychologies of space. In Spatial Information Theory A Theoretical Basis for GIS, Andrew U. Frank and Irene Campari (Eds.). Springer Berlin Heidelberg, 312-321. https://doi.org/10.1007/3-54057207-4_21

[26] Clark C Presson and Daniel R Montello. 1994. Updating after Rotational and Translational Body Movements: Coordinate Structure of Perspective Space. Perception 23, 12 (1994), 1447-1455. https://doi.org/10.1068/p231447

[27] Eric D Ragan, Siroberto Scerbo, Felipe Bacim, and Doug A Bowman. 2016. Amplified Head Rotation in Virtual Reality and the Effects on 3D Search, Training Transfer, and Spatial Orientation. IEEE Transactions on Visualization and Computer Graphics 23, 8 (2016), 1880-1895. https://doi.org/10.1109/TVCG.2016.2601607

[28] Kasra Rahimi, Colin Banigan, and Eric D. Ragan. 2020. Scene Transitions and Teleportation in Virtual Reality and the Implications for Spatial Awareness and Sickness. IEEE Transactions on Visualization and Computer Graphics 26 (2020), 2273-2287. https://doi.org/10.1109/TVCG.2018.2884468

[29] Lisa Rebenitsch and Charles Owen. 2016. Review on Cybersickness in Applications and Visual Displays. Virtual Reality 20, 2 (June 2016), 101-125. https://doi.org/10.1007/s10055-016-0285-9

[30] Bernhard E Riecke, Douglas W Cunningham, and Heinrich H Bülthoff. 2007. Spatial updating in virtual reality: the sufficiency of visual information. Psychological research 71, 3 (2007), 298-313. https://doi.org/10.1007/s00426-006-0085-z

[31] S. P. Sargunam, K. R. Moghadam, M. Suhail, and E. D. Ragan. 2017. Guided head rotation and amplified head rotation: Evaluating semi-natural travel and viewing techniques in virtual reality. In 2017 IEEE Virtual Reality (VR). 19-28. https://doi.org/10.1109/VR.2017.7892227

[32] Sarah Sharples, Sue Cobb, Amanda Moody, and John R Wilson. 2008. Virtual reality induced symptoms and effects (VRISE): Comparison of head mounted display (HMD), desktop and projection display systems. Displays 29, 2 (2008), 58-69. https://doi.org/10.1016/j.displa.2007.09.005

[33] Daniel R Trindade and Alberto B Raposo. 2011. Improving 3D navigation in multiscale environments using cubemap-based techniques. In Proceedings of the 2011 ACM Symposium on Applied Computing. 1215-1221.

[34] Martin Usoh, Kevin Arthur, Mary C Whitton, Rui Bastos, Anthony Steed, Mel Slater, and Frederick P Brooks Jr. 1999. Walking > walking-in-place $>$ flying, in virtual environments. In Proceedings of the 26th Annual Conference on Computer Graphics and Interactive Techniques. 359-364. https://doi.org/10.1145/311535. 311589

[35] Steven Vi, Tiago Silva da Silva, and Frank Maurer. 2019. User Experience Guidelines for Designing HMD Extended Reality Applications. In IFIP Conference on Human-Computer Interaction. 319-341. https://doi.org/10.1007/978-3-030-29390$1 \_18$

[36] Tìm Weissker, André Kunert, Bernd Froehlich, and Alexander Kulik. 2018. Spatial updating and simulator sickness during steering and jumping in immersive virtual environments. In 2018 IEEE Conference on Virtual Reality and 3D User Interfaces (VR). 97-104. https://doi.org/10.1109/VR.2018.8446620 